\title{
NMPC suboptimality estimates for sampled-data continuous systems
}

\author{
Lars Grüne, Marcus von Lossow, and Karl Worthmann \\ Mathematical Institute, University of Bayreuth, 95440 Bayreuth, Germany \\ lars.gruene, marcus.vonlossow, karl.worthmann@uni-bayreuth.de
}

Summary. In this paper we consider unconstrained model predictive control (MPC) schemes and investigate known stability and performance estimates with respect to their applicability in the context of sampled-data systems. To this end, we show that these estimates become rather conservative for sampling periods tending to zero which is, however, typically required for sampled-data systems in order to inherit the stability behavior of their continuous-time counterparts. We introduce a growth condition which allows for incorporating continuity properties in the MPC performance analysis and illustrate its impact - especially for fast sampling.

\section{Introduction}

In order to deal with optimal control problems on an infinite horizon we use model predictive control (MPC). This method relies on an iterative online solution of finite horizon optimal control problems. To this end, a performance criterion is optimized over the predicted trajectories of the system. The stability and performance analysis of linear and nonlinear MPC schemes has attracted considerable attention during the last years, cf. [2, 9].

Here we consider unconstrained nonlinear MPC (NMPC) schemes which are frequently used in industrial applications, cf. [8]. These incorporate neither additional terminal constraints nor terminal costs in the finite horizon problems in order to enforce stability properties. Nevertheless, a stability analysis - based on a controllability assumption - is possible and given in $[3,5]$.

In the present paper we focus on sampled-data continuous systems. Typically, these require sufficiently fast sampling in order to preserve their stability properties, cf. [7]. However, the direct application of [3, 5] leads to very pessimistic performance bounds, cf. Section 4. In order to compensate for this drawback we incorporate a growth condition which reflects properties of the considered sampled-data systems in the ensuing section. Finally, we investigate qualitative and quantitative effects related to the proposed condition. 


\section{Setup and Preliminaries}

We consider a nonlinear discrete time control system given by

$$
x(n+1)=f(x(n), u(n)), \quad x(0)=x_{0}
$$

with $x(n) \in X$ and $u(n) \in U$ for $n \in \mathbb{N}_{0}$. Here the state space $X$ and the control value space $U$ are arbitrary metric spaces. We denote the space of control sequences $u: \mathbb{N}_{0} \rightarrow U$ by $\mathcal{U}$ and the solution trajectory for given $u \in \mathcal{U}$ by $x_{u}(\cdot)$. A typical class of such discrete time systems are sampled-data systems induced by a controlled — finite or infinite dimensional — differential equation with sampling period $T>0$, see Section 4 for details.

Our goal consists of minimizing the infinite horizon cost $J_{\infty}\left(x_{0}, u\right)=$ $\sum_{n=0}^{\infty} l\left(x_{u}(n), u(n)\right)$ with running cost $l: X \times U \rightarrow \mathbb{R}_{0}^{+}$by a static state feedback control law $\mu: X \rightarrow U$ which is applied according to the rule $x_{\mu}(0)=x_{0}$,

$$
x_{\mu}(n+1)=f\left(x_{\mu}(n), \mu\left(x_{\mu}(n)\right)\right) .
$$

We denote the optimal value function for this problem by $V_{\infty}\left(x_{0}\right):=\inf _{u \in \mathcal{U}}$ $J_{\infty}\left(x_{0}, u\right)$. Since infinite horizon optimal control problems are in general computationally intractable, we use a receding horizon approach in order to compute an approximately optimal controller. To this end, we consider the finite horizon functional

$$
J_{N}\left(x_{0}, u\right)=\sum_{n=0}^{N-1} l\left(x_{u}(n), u(n)\right)
$$

with optimization horizon $N \in \mathbb{N}_{\geq 2}$ inducing the optimal value function

$$
V_{N}\left(x_{0}\right)=\inf _{u \in \mathcal{U}} J_{N}\left(x_{0}, u\right) .
$$

By solving this finite horizon optimal control problem we obtain $N$ control values $u^{*}(0), u^{*}(1), \ldots, u^{*}(N-1)$ which depend on the state $x_{0}$. Implementing the first element of this sequence, i.e., $u^{*}(0)$, yields a new state $x(1)$. Iterative application of this construction provides a control sequence on the infinite time interval. We obtain a closed loop representation by applying the map $\mu_{N}: X \rightarrow U$ which is given in Definition 1 as a static state feedback law.

Definition 1. For $N \in \mathbb{N}_{\geq 2}$ we define the MPC feedback law $\mu_{N}\left(x_{0}\right):=$ $u^{\star}(0)$, where $u^{\star}$ is a minimizing control for (4) with initial value $x_{0}$.

Remark 1. For simplicity of exposition we assume that the infimum in (4) is a minimum, i.e., that a minimizing control sequence $u^{*}$ exists.

In this paper we consider the conceptually simplest MPC approach imposing neither terminal costs nor terminal constraints. In order to measure the suboptimality degree of the MPC feedback for the infinite horizon problem we define

$$
V_{\infty}^{\mu}\left(x_{0}\right):=\sum_{n=0}^{\infty} l\left(x_{\mu}(n), \mu\left(x_{\mu}(n)\right)\right) .
$$




\section{Controllability and performance bounds}

In this section we introduce an exponential controllability assumption and deduce several consequences for our optimal control problem. In order to facilitate this relation we will formulate our basic controllability assumption not in terms of the trajectory but in terms of the running cost $l$ along a trajectory. To this end, we define $l^{\star}(x):=\min _{u \in U} l(x, u)$.

Property 1. Assume exponential controllability with overshoot bound $C \geq 1$ and decay rate $\sigma \in(0,1)$, i.e., for each $x_{0} \in X$ there exists a control function $u_{x_{0}} \in \mathcal{U}$ satisfying the estimate

$$
l\left(x_{u_{x_{0}}}(n), u_{x_{0}}(n)\right) \leq C \sigma^{n} l^{\star}\left(x_{0}\right) \quad \text { for all } n \in \mathbb{N}_{0} .
$$

Based on Property 1 and Bellman's optimality principle an optimization problem is derived in [3] whose solution, which depends on the optimization horizon $N$, coincides with the parameter $\alpha_{N}$ in the relaxed Lyapunov inequality $V_{N}\left(f\left(x, \mu_{N}(x)\right)\right) \leq V_{N}(x)-\alpha_{N} l\left(x, \mu_{N}(x)\right)$. As a consequence the estimate

$$
\alpha_{N} V_{\infty}(x) \leq \alpha_{N} V_{\infty}^{\mu_{N}}(x) \leq V_{N}(x)
$$

holds for all $x \in X$. Hence, $\alpha_{N}$ specifies a suboptimality degree. For details we refer to [1]. Since we focus on the stability behavior of systems satisfying (5), i.e. exponential controllability, it is possible to calculate this performance index $\alpha_{N}$ explicitly, cf. [5, section 5].

Theorem 1. Assume Property 1 and let the optimization horizon $N$ be given. Then we obtain for the suboptimality degree $\alpha_{N}$ from (6) the formula

$$
\alpha_{N}=1-\frac{\left(\gamma_{N}-1\right) \prod_{i=2}^{N}\left(\gamma_{i}-1\right)}{\prod_{i=2}^{N} \gamma_{i}-\prod_{i=2}^{N}\left(\gamma_{i}-1\right)} \quad \text { with } \quad \gamma_{i}:=C \sum_{n=0}^{i-1} \sigma^{n}=C \frac{1-\sigma^{i}}{1-\sigma}
$$

Remark 2. Theorem 1 is generalizable to functionals including an additional weight on the final term. This may enhance the stability behavior of the underlying system significantly. Moreover, it remains valid for more general controllability assumptions, for instance, finite time controllability with linear overshoot, cf. [5, Sections 5 and 8] for details.

Remark 3. Theorem 1 is also applicable in the context of networked control systems which require the implementation of more than only the first element of the obtained sequence of control values, cf. [6] for details. 


\section{Sampled-data systems and arbitrary fast sampling}

Given a continuous time control system governed by the differential equation $\dot{\varphi}=g(\varphi(t), \tilde{u}(t))$, we assume exponential controllabilty, i.e., that for each $x_{0} \in X$ there exists a control function $\tilde{u}_{x_{0}}(\cdot)$ such that

$$
l\left(\varphi\left(t ; x_{0}, \tilde{u}_{x_{0}}\right), \tilde{u}_{x_{0}}(t)\right) \leq C e^{-\lambda t} l^{*}\left(x_{0}\right)
$$

holds almost everywhere for given overshoot $C \geq 1$ and decay rate $\lambda>0$. Here $\varphi\left(t ; x_{0}, \tilde{u}\right)$ denotes the solution of the respective control system. In order to analyze the stability behavior, we define the discrete time system (1) by $f(x, u):=\varphi(T ; x, \tilde{u})$ with discretization parameter $T>0$. Consequently, the assumed exponential controllability of the continuous time system implies (5) in the discrete time setting, i.e., Property 1 with $\sigma=e^{-\lambda T}$ for an appropriately chosen control value space. Moreover, we fix the continuous time optimization interval $\left[0, t_{F}\right)$ which corresponds to an optimization horizon of length $N=$ $t_{F} / T$ in the discrete time setting.

A typical representative of this class are sampled-data systems with sampling period $T_{0}:=T$ and piecewise constant control, i.e., $\tilde{u}(t)=u$ for all $t \in\left[0, T_{0}\right)$. However, sampled-data systems require sufficiently fast sampling in order to inherit the stability behavior from (8), cf. [7]. Consequently, it may be necessary to increase the sampling rate, i.e., using smaller sampling periods. In this section we focus on effects caused by this adjustment. Thus, we reduce the discretization parameter of the discrete time model along with the sampling rate of the sampled-data system in consideration.

In order to investigate this issue systematically, we consider the sequence of sampling periods $T_{0}, T_{0} / 2, T_{0} / 4, \ldots$, i.e., $T_{k}=2^{-k} T_{0}$. This determines the optimization horizons $N_{0}, 2 N_{0}, 4 N_{0}, \ldots$, i.e. $N_{k}=2^{k} N_{0}$, for the discrete time system because we have fixed the optimization interval $\left[0, t_{F}\right)$ and coupled the discretization parameter with the sampling period. The corresponding decay rate from (8) is $\sigma_{k}=e^{-\lambda T_{k}}$, cf. Figure 1 on the left. Hence, we consider the sequence

$$
\left(T_{k}, N_{k}, \sigma_{k}\right)_{k \in \mathbb{N}_{0}}=\left(2^{-k} T_{0}, 2^{k} N_{0}, e^{-\lambda T_{k}}\right)_{k \in \mathbb{N}_{0}}
$$

of parameter combinations consisting of sampling period, optimization horizon, and decay rate. Note that the interval $\left[0, T_{k}\right)$ on which the first element of the calculated control value sequence is applied scales down as well.

We require the following definition.

Definition 2. Let $C \geq 1, \lambda>0$, and $T_{0}>0$ be given. In addition, we set $\sigma_{k}:=e^{-\lambda\left(2^{-k} T_{0}\right)}$. Then we define

$$
\gamma_{i}^{k}:=\sum_{n=0}^{i-1} C e^{-\lambda\left(2^{-k} T_{0}\right) n}=C \sum_{n=0}^{i-1} \sigma_{k}^{n}=\frac{C\left(1-\sigma_{k}^{i}\right)}{1-\sigma_{k}} .
$$

Remark 4. Note that we use an additional index in order to indicate the dependence of $\gamma_{i}^{k}$ on $\sigma_{k}$. For $k=0$ we obtain exactly $\gamma_{i}$ from (7). Moreover, the relation $\sigma_{k}=\sqrt{\sigma_{k-1}}=\sigma_{0}^{2^{-k}}$ holds. 
Theorem 2 shows that the suboptimality estimates from Theorem 1 become arbitrarily bad for sampling periods tending to zero, cf. Figure 1. In order to compensate this drawback for sampled-data continuous systems we introduce an appropriate condition in the ensuing section.
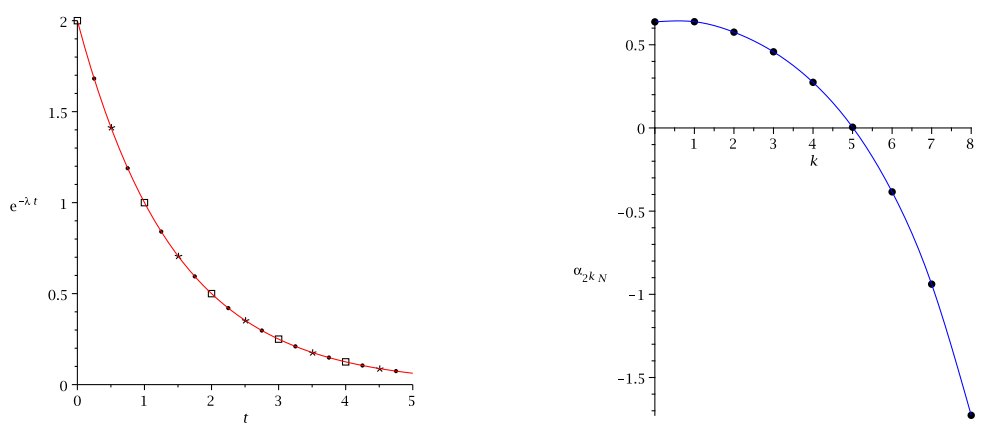

Fig. 1. Visualization of the bounds induced by our controllability assumption for $\left(2^{-k} T_{0}, 2^{k} N_{0}, e^{-\lambda\left(2^{-k} T_{0}\right)}\right)_{k \in \mathbb{N}_{0}}$ with $T_{0}=1, N_{0}=8, \lambda=-\ln (1 / 2)$, and $C=2$ for $k=0,1,2(\square, *, \cdot)$ on the left. On the right we depict the suboptimality estimates $\alpha_{N_{k}}^{k}, k=0,1,2, \ldots, 8$, from Theorem 2 for this sequence.

Theorem 2. Assume (8) and let $N_{0}:=N \in \mathbb{N}_{\geq 2}, T_{0}>0$ be given. Then the suboptimality bounds corresponding to the sequence $\left(T_{k}, N_{k}, \sigma_{k}\right)_{k \in \mathbb{N}_{0}}=$ $\left(2^{-k} T_{0}, 2^{k} N_{0}, e^{-\lambda\left(2^{-k} T_{0}\right)}\right)_{k \in \mathbb{N}_{0}}$ diverge to $-\infty$, i.e.,

$$
\alpha_{N_{k}}^{k}=1-\frac{\left(\gamma_{N_{k}}^{k}-1\right) \prod_{i=2}^{N_{k}}\left(\gamma_{i}^{k}-1\right)}{\prod_{i=2}^{N_{k}} \gamma_{i}^{k}-\prod_{i=2}^{N_{k}}\left(\gamma_{i}^{k}-1\right)} \longrightarrow-\infty \quad \text { for } \quad k \rightarrow \infty
$$

with $\gamma_{i}^{k}$ from Definition 2 .

Proof. Since $\prod_{i=2}^{2^{k} N} \gamma_{i}^{k} \geq \prod_{i=2}^{2^{k} N}\left(\gamma_{i}^{k}-1\right) \geq 0$ proving the assertion follows from

$$
0 \leq \frac{1}{\gamma_{2^{k} N}^{k}-1} \cdot \prod_{i=2}^{2^{k} N} \frac{\gamma_{i}^{k}}{\gamma_{i}^{k}-1} \stackrel{k \rightarrow \infty}{\longrightarrow} 0 .
$$

In order to estimate (12) we establish the inequalities

$$
\frac{1}{\gamma_{2^{k} N}^{k}-1} \leq \frac{1-\sigma_{k}}{C_{1}} \quad \text { and } \quad \prod_{i=2}^{2^{k} N} \frac{\gamma_{i}^{k}}{\gamma_{i}^{k}-1} \leq C_{0}\left(2^{1 / C}\right)^{k}
$$

with $C_{0}:=\sigma_{0}^{-N / C} \prod_{i=2}^{N} \frac{i C}{i C-1}$ and $C_{1}:=C\left(1-\sigma_{0}^{N}\right)-1+\sigma_{0}$. Note that $C_{0}$ and $C_{1}$ do not depend on $k$. The first inequality is directly implied by Definition 2. In order to show the second we prove the inequality 


$$
\frac{\gamma_{i}^{k}}{\gamma_{i}^{k}-1}=\frac{C}{C-1+\sigma_{k}} \frac{\left(1-\sigma_{k}^{i}\right)\left(C-1+\sigma_{k}\right)}{C-1+\sigma_{k}-C \sigma_{k}^{i}} \leq \frac{C}{C-1+\sigma_{k}} \cdot \frac{i C}{i C-1}
$$

which is equivalent to $i \sigma_{k}^{i} C\left(1-\sigma_{k}\right) \leq\left(C-1+\sigma_{k}\right)\left(1-\sigma_{k}^{i}\right), k \in \mathbb{N}_{0}$ and $i \in \mathbb{N}_{\geq 1}$. Since $C \sigma_{k} /\left(C-1+\sigma_{k}\right) \leq 1$ this is shown by $i \sigma_{k}^{i-1} \leq \sum_{n=0}^{i-1} \sigma_{k}^{n}=$ $\left(1-\sigma_{k}^{i}\right) /\left(1-\sigma_{k}\right)$. Moreover, we require the inequality

$$
\left(\frac{C}{C-1+\sigma_{k}}\right)^{2^{k} N} \leq \sigma_{0}^{-N / C}
$$

which is - in consideration of Definition 2 - equivalent to $f\left(\sigma_{k}\right):=C-C \sigma_{k}^{1 / C}$ $1+\sigma_{k} \geq 0$. However, since $f(0)=C-1 \geq 0$ and $f(1)=0$ the inequality $f^{\prime}\left(\sigma_{k}\right)=1-\sigma_{k}^{-(C-1) / C} \leq 0$ implies (14).

Hence, taking into account that the factor $C /\left(C-1+\sigma_{k}\right)$ is independent of the control variable $i$ and applying the two deduced estimates leads to

$$
\prod_{i=2}^{2^{k} N} \frac{\gamma_{i}^{k}}{\gamma_{i}^{k}-1}<\sigma_{0}^{-N / C} \cdot \prod_{i=2}^{2^{k} N} \frac{i C}{i C-1}=C_{0} \prod_{j=0}^{k-1}\left(\prod_{i=2^{j} N+1}^{2^{j+1} N} \frac{i C}{i C-1}\right)
$$

for $k \in \mathbb{N}_{0}$. Thus, it suffices to estimate the expression in brackets uniformly from above by $2^{1 / C}$ for $j \in \mathbb{N}_{\geq 0}$ in order to show (13).

In the following, we use the functional equation, i.e., $\Gamma(x+1)=x \Gamma(x)$ and $\Gamma(1)=1$, for the gamma function $\Gamma(\cdot)$ which is connected to the beta function $B(\cdot, \cdot)$ via the formula

$$
B(x, y)=\frac{\Gamma(x) \Gamma(y)}{\Gamma(x+y)},
$$

cf. $[11$, p.442]. Moreover, we require the equation

$$
B(p, p+s)=\frac{B(p, p)}{2^{s}}\left(1+\frac{s(s-1)}{2(2 p+1)}+\frac{s(s-1)(s-2)(s-3)}{2 \cdot 4 \cdot(2 p+1) \cdot(2 p+3)}+\ldots\right)
$$

which holds for $p>0, p+s>0$ according to [10, p.262] in order to estimate the term in brackets from (15) as follows

$$
\begin{aligned}
\prod_{i=2^{k} N+1}^{2^{k+1} N} \frac{i C}{i C-1} & =\prod_{i=2^{k} N+1}^{2^{k+1} N} \frac{i}{i-\frac{1}{C}}=\frac{\left(2^{k+1} N\right) !}{\left(2^{k} N\right) !}\left(\prod_{i=2^{k} N+1}^{2^{k+1} N} i-\frac{1}{C}\right)^{-1} \\
& =\frac{\Gamma\left(2^{k+1} N+1\right)}{\Gamma\left(2^{k} N+1\right)} \cdot \frac{\Gamma\left(2^{k} N+1-\frac{1}{C}\right)}{\Gamma\left(2^{k+1} N+1-\frac{1}{C}\right)} \\
& \stackrel{(16)}{=} \frac{B\left(2^{k} N, 2^{k} N+\frac{C-1}{C}\right)}{B\left(2^{k} N, 2^{k} N+1\right)} \\
& \stackrel{(17)}{=} 2^{1 / C}\left(1+\frac{s(s-1)}{2(2 p+1)}+\frac{s(s-1)(s-2)(s-3)}{2 \cdot 4 \cdot(2 p+1) \cdot(2 p+3)}+\ldots\right)
\end{aligned}
$$


with $s=(C-1) / C \in[0,1)$ and $p=2^{k} N$. Since $s \in[0,1)$ the term in brackets is less or equal to one. Hence, we obtain the desired estimate (13).

Thus, it suffices to show $\left(2^{1 / C}\right)^{k}\left(1-\sigma_{k}\right) \rightarrow 0$ as $k$ approaches infinity in order to complete the proof. To this aim, we define $a_{k}:=\left(2^{1 / C}\right)^{k}\left(1-\sigma_{k}\right)$ and show that the quotient $a_{k+1} / a_{k}$ converges to $2^{1 / C} / 2$ for $k \rightarrow \infty$ :

$$
\frac{a_{k+1}}{a_{k}}=\frac{1-\sigma_{k+1}}{1-\sigma_{k}} 2^{1 / C}=\frac{\left(1-\sigma_{k+1}\right) 2^{1 / C}}{\left(1-\sigma_{k+1}\right)\left(1+\sigma_{k+1}\right)}=\frac{2^{1 / C}}{1+\sigma_{0}^{2^{-(k+1)}}} \stackrel{k \rightarrow \infty}{\longrightarrow} 2^{1 / C} / 2 .
$$

Thus, there exists $k^{*}$ such that the considered quotient $a_{k+1} / a_{k}$ is less or equal $\theta:=\left(2+2^{1 / C}\right) / 4<1$ for all $k \geq k^{*}$. This implies the convergence of $a_{k}=2^{1 / C}\left(1-\sigma_{k}\right)$ to zero for $k$ approaching infinity.

\section{Growth condition and analytic formula}

Although the estimate stated in Theorem 1 is strict for the whole class of systems satisfying the assumed controllability condition, cf. [3, Theorem 5.3], it may be conservative for subsets of this class. For instance, for sampleddata continuous time systems the difference between $x(n+1)$ and $x(n)$ is usually of order $\mathcal{O}(T)$, a property which is not reflected in the optimization problem on which Theorem 1 is based on. Neglecting this leads to very pessimistic estimates if the sampling period $T$ tends to 0 and the continuous time optimization horizon $H=\left[0, t_{F}\right)$ is fixed, cf. Section 4 .

In order to compensate for this drawback, we incorporate a growth condition in our suboptimality estimate.

Property 2. For each $x_{0} \in X$ there exists a control function $\tilde{u}_{x_{0}}(\cdot) \in \mathcal{U}$ such that

$$
l\left(\varphi\left(t ; x_{0}, \tilde{u}_{x_{0}}\right), \tilde{u}_{x_{0}}(t)\right) \leq e^{L_{c} t} l^{*}\left(x_{0}\right) \quad \text { for all } t \geq 0
$$

with constant $L_{c}>0$ which is independent of the chosen state $x_{0}$. Let a discretization parameter $T>0$ be given and define the discrete time system by $f(x, \tilde{u})=\varphi(T ; x, \tilde{u})$ with an appropriately chosen control value space $U$. Then - as a consequence from (18) - the inequality

$$
J_{N-k}\left(x_{\tilde{u}_{x_{0}}}(k), \tilde{u}_{x_{0}}(k+\cdot)\right) \leq l^{*}\left(x_{\tilde{u}_{x_{0}}}(k)\right) \sum_{n=0}^{N-k-1} L^{n}
$$

holds with $L=e^{L_{c} T}>1$ for $k=0, \ldots, N-1$.

In combination with our controllability property (8) this leads to the definition

$$
\gamma_{i}:=\min \left\{C \sum_{n=0}^{i-1} \sigma^{n}, \sum_{n=0}^{i-1} L^{n}\right\}
$$



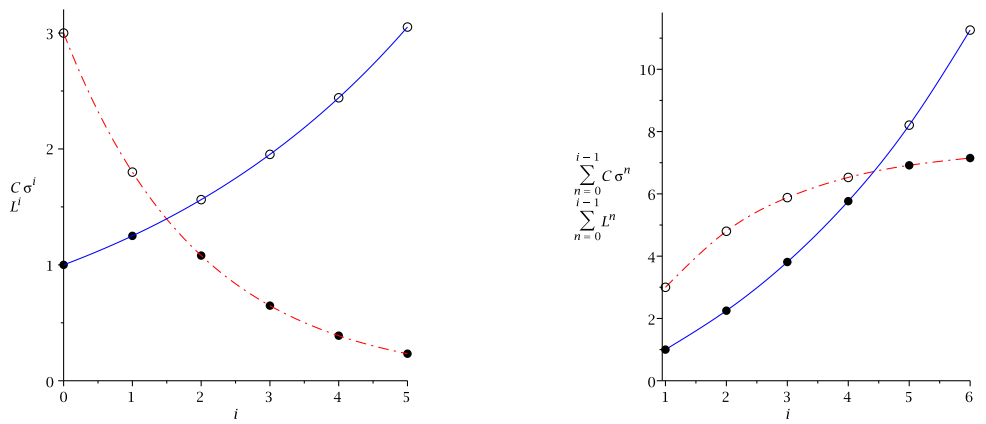

Fig. 2. Visualization of the bounds induced by our controllability assumption (dashed-dotted line) and our growth condition (solid line) for $C=3, \sigma=3 / 5$, and $L=5 / 4$. Each time the minimum is marked with solid circles. The solid circles on the right coincide with $\gamma_{i}$ from (19)

with $\sigma:=e^{-\lambda T}$ and $L$ from Property 2 . Thus, we obtain tighter bounds with respect to the stage costs where the introduced growth condition is applicable in contrast to $\gamma_{i}$ from (7), cp. Figure 2.

Theorem 1 remains valid if we substitute the definition of $\gamma_{i}$ in (7) by (19).

Theorem 3. Assume exponential controllability and our growth condition, i.e., Properties 1 and 2, with parameters $\sigma \in(0,1), C \geq 1$, and $L \geq 1$ then we obtain for given optimization horizon $N$ Formula (7) with $\gamma_{i}$ from (19).

Proof. Sifting through the proof of Theorem 1 one notices that changing the definition of $\gamma_{i}$ to (19) does not affect the part of the proof in which (7) is established as the solution of the relaxed optimization problem, cf. [5, Problem 5.3]. However, we have to show the inequality

$$
\left(\gamma_{2}-1\right) \prod_{i=3}^{N-j+1}\left(\gamma_{i}-1\right) \geq\left(\gamma_{N-j+1}-\gamma_{N-j}\right) \prod_{i=2}^{N-j} \gamma_{i}, \quad j=1, \ldots, N-2
$$

which implies [5, Inequality (5.8)] for $m=1, \omega=1$ and - as a consequence ensures that Formula (7) provides the solution of the respective optimization problem.

Moreover, note that there exists exactly one index $i^{\star} \in \mathbb{N}_{\geq 1}$ such that $\gamma_{i^{\star}}=\sum_{n=0}^{i^{\star}-1} L^{n}$ and $\gamma_{i^{\star}+1}<\sum_{n=0}^{i^{\star}} L^{n} \cdot n^{\star} \geq N-j+1$ corresponds to $C:=$ $L \geq 1$ and $\sigma:=1$. However, since [5] shows the desired inequality for arbitrary $\sigma \in(0,1)$ this situation is covered. $n^{\star}=N-j$ is also trivial, since we may estimate $\gamma_{N-j+1} \leq \sum_{n=0}^{N-j} L^{n}$. Thus, $\gamma_{N-j+1}=\gamma_{N-j}+C \sigma^{N-j}=C \sum_{n=0}^{N-j} \sigma^{n}$ holds. We rewrite the above inequality as

$$
(C-1) \prod_{i=2}^{N-j}\left(\gamma_{i}-1\right)+C \prod_{i=2}^{N-j}\left(\gamma_{i}-1\right) \sum_{n=1}^{N-j} \sigma^{n} \geq C \sigma^{N-j} \prod_{i=2}^{N-j} \gamma_{i} .
$$


Consequently, it suffices to show $\prod_{i=2}^{N-j}\left(\gamma_{i}-1\right) \sum_{n=1}^{N-j} \sigma^{n} \geq \sigma^{N-j} \prod_{i=2}^{N-j} \gamma_{i}$ which can be done by induction. The induction start $j=N-2$ is $\left(\gamma_{2}-\right.$ 1) $\left(\sigma+\sigma^{2}\right) \geq \sigma^{2} \gamma_{2}$ or equivalently $\sigma\left(\gamma_{2}-(1+\sigma)\right) \geq 0$ which holds due to the definition of $\gamma_{2}$. The induction step from $j+1 \rightsquigarrow j$ holds since the desired inequality may be written as

$$
\prod_{i=2}^{N-\bar{j}}\left(\gamma_{i}-1\right)\left[\sigma \gamma_{N-j}-\sum_{n=1}^{N-j} \sigma^{n}\right]+\sigma \gamma_{N-j}\left[\prod_{i=2}^{N-\bar{j}}\left(\gamma_{i}-1\right) \sum_{n=1}^{N-\bar{j}} \sigma^{n}-\sigma^{N-\bar{j}} \prod_{i=2}^{N-\bar{j}} \gamma_{i}\right] \geq 0 .
$$

with $\bar{j}:=j+1$.

Remark 5. Conditions which guarantee Property 2 can be found in [4].

\section{Numerical Examples}

We have observed that sampling periods tending to zero cause serious problems in applying our estimates from Theorem 1, cf. Figure 1. In order to compensate for this drawback we introduced Property 2 for sampled-data continuous time systems and generalized our results to this setting, cf. Theorem 3. This justifies the application of Formula (7) in consideration of the imposed growth condition and enables us to analyze its impact.

Again, we fix the continuous time optimization interval $\left[0, t_{F}\right)$ and consider sequence (9). However, we assume - in addition to (8) - Property 2. As a consequence, we obtain the suboptimality bounds from Formula (7) with

$$
\gamma_{i}^{k}:=\min \left\{C \sum_{n=0}^{i-1} \sigma_{k}^{n}, \sum_{n=0}^{i-1} L_{k}^{n}\right\}
$$

with $\sigma_{k}:=e^{-\lambda T_{k}}=e^{-\lambda\left(2^{-k} T_{0}\right)}$ and $L_{k}:=e^{L_{c} T_{k}}=e^{L_{c}\left(2^{-k} T_{0}\right)}$. As shown by Figure 3 , our continuity condition counteracts occurring problems in connection with arbitrary fast sampling.

Next, we consider quantitative effects related to Property 2. Since the overshoot $C$ has been proven to be the decisive parameter in order to establish stability, cf. [3, section 6], [5, section 6$]$, we investigate its sensitivity to changes in the growth constant $L$. To this aim, we fix the decay rate $\sigma=0.7$. Our goal consists of determining the maximal overshoot $C$ which allows for guaranteeing stability for the whole class of systems, i.e., $\alpha_{N} \geq 0$, for a given optimization horizon $N$. Neglecting our growth condition yields the values $1.8189,2.0216,2.2208$ for $N=8,10$, and 12 respectively. Whereas Figure 3 shows that Property 2 allows for significantly larger values for $C$. The impact of our growth condition remains basically the same for $\alpha_{N}>0$, i.e., if we do not only aim at ensuring stability, but also set performance specification on our calculated NMPC-Feedback.

Hence, Property 2 allows for calculating tighter bounds, i.e. larger $\alpha_{N}$ values, and consequently a more accurate characterization of the system's behavior. 

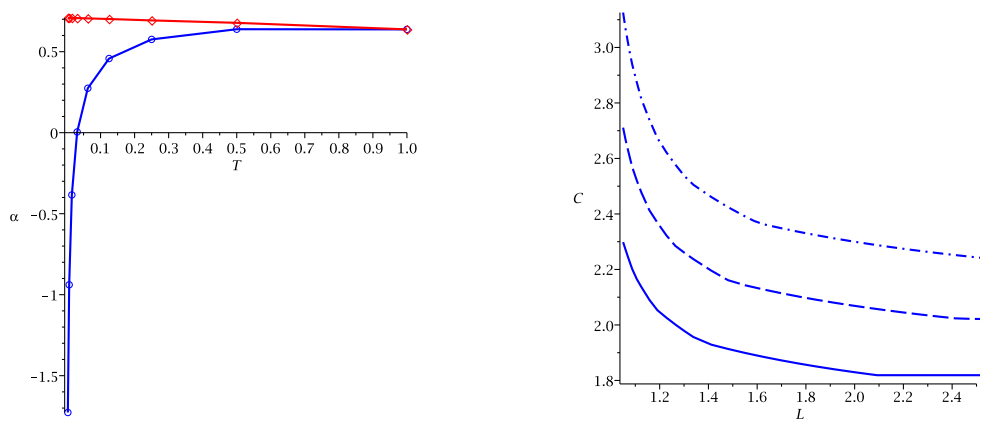

Fig. 3. On the left we depict the suboptimality estimates obtained from (7) with $\gamma_{i}^{k}$ from (20) with $(\diamond)$ and without (o) our growth condition (2) in dependence on the sampling period $T$. The parameters are the same as used for Figure 1. On the right the maximal feasible overshoot $C$ in dependence of our growth constant $L$ is presented for given overshoot $\sigma$ and optimization horizon $N=8$ (solid), 10 (dashed), and 12 (dash-dotted) respectively for which Theorem 3 guarantees stability, i.e., $\alpha_{N} \geq 0$.

\section{References}

1. Altmüller N, Grüne L, Worthmann K (2009), Performance of NMPC schemes without stabilizing terminal constraints, submitted to BFG 09.

2. Allgöwer F, Zheng A, eds. (2000), Nonlinear model predictive control, Birkhäuser, Basel.

3. Grüne L (2009) Analysis and design of unconstrained nonlinear MPC schemes for finite and infinite dimensional systems, SIAM J. Control Optim., 48, pp. $1206-1228$.

4. Grüne L, von Lossow M, Pannek J, Worthmann K (2010), MPC: implications of a growth condition on exponentially controllable systems, submitted.

5. Grüne L, Pannek J, Seehafer M, Worthmann K (2009), Analysis of unconstrained nonlinear MPC schemes with time varying control horizon, submitted.

6. Grüne L, Pannek J, Worthmann K (2009), A networked unconstrained nonlinear MPC scheme, Proceedings of the European Control Conference 2009, Budapest, Hungary, pp. 371-376.

7. Nešić D, Teel A R (2004), A framework for stabilization of nonlinear sampleddata systems based on their approximate discrete-time models, IEEE Trans. Automat. Control, 49 (7), pp. 1103-1122.

8. Qin S, Badgwell T (2003), A survey of industrial model predictive control technology, Control Engineering Practice, 11, pp. 733-764.

9. Rawlings J B, Mayne D Q (2009), Model Predictive Control: Theory and Design, Nob Hill Publishing, Madison.

10. Whittaker E T, Watson G N (1929), A course of Modern Analysis, Cambridge University Press, New York, 4th edition.

11. Zorich V A (2004), Mathematical Analysis II, Springer, Berlin Heidelberg. 


\section{Index}

controllability, 3

exponential controllability, 3

growth condition, 7

model predictive control, 1

nonlinear model predictive control, 1

optimal value function, 2 performance bounds, 3

receding horizon, 2

relaxed Lyapunov inequality, 3

sampled-data systems, 4

sampling rate, 4

stability analysis, 1

suboptimality, 3 